\title{
Structure and optical properties of amorphous lead-germanate films developed by pulsed-laser deposition
}

\author{
M. Dussauze, A. Giannoudakos, L. Velli, C. P. E. Varsamis, \\ M. Kompitsas, and E. I. Kamitsos ${ }^{\text {a) }}$ \\ Theoretical and Physical Chemistry Institute, National Hellenic Research Foundation, \\ 48 Vassileos Constantinou Avenue, 11635 Athens, Greece
}

(Received 2 April 2007; accepted 1 June 2007; published online 19 July 2007)

\begin{abstract}
Lead-germanate materials are attractive systems for photonics applications. In this context, amorphous lead-germanate films were grown by pulsed-laser deposition at different substrate temperatures and oxygen pressures using a glassy target of composition $0.4 \mathrm{PbO}-0.6 \mathrm{GeO}_{2}$. Optical and infrared measurements showed that the substrate temperature has a strong influence on the optical quality and stability of the deposited films. An accurate characterization of films was achieved by comparing experimental and simulated transmittance spectra in the infrared, and allowed to probe the structural evolution and variations in composition as a function of oxygen pressure. The results showed that the difference in reactivity of lead and germanium toward oxygen in the laser-produced plasma allows for composition adjustments in the lead-germanate films by varying the oxygen pressure in the deposition chamber. (C) 2007 American Institute of Physics. [DOI: $10.1063 / 1.2752503$ ]
\end{abstract}

\section{INTRODUCTION}

The use of planar waveguide configurations has received great attention for the fabrication of photonic devices such as planar light circuits that can combine all passive and active functions in the same chip. Glassy thin films appear to be serious candidates in this domain of applications as they can exhibit low losses, ${ }^{1-3}$ high amplification by rare earth doping, ${ }^{4-6}$ and efficient second order nonlinear optical properties induced by thermal poling. ${ }^{7-9}$ In this family of amorphous materials, lead-germanate glasses are attractive systems because of their large $\chi^{(3)}$ nonlinearity and significant photosensitivity that may lead to efficient optical grating and feedback structures. ${ }^{10,11}$

Glass thin films can be prepared by different deposition techniques, among which pulsed-laser deposition (PLD) is a relatively quick and simple technique. Also, it is a suitable method for the deposition of materials with complex compositions. However, various deposition techniques may lead to glassy thin films with physicochemical properties presenting differences with respect to those of bulk materials with the same composition. For example, early studies on silicon dioxide, $\mathrm{SiO}_{2}$, have shown that this is indeed the case for thin films prepared by evaporation, ${ }^{12} \mathrm{dc}$ sputtering, ${ }^{13}$ as well as by rf-magnetron sputtering. ${ }^{14}$ As a consequence, a key step in the development of glassy thin films is to establish the effect of preparation conditions on thin film composition, structure, and properties.

In an earlier study ${ }^{15}$ lead-germanate thin films were developed by PLD at various oxygen pressures from bulk glassy targets of composition $x \mathrm{PbO}-(1-x) \mathrm{GeO}_{2}$ with $x$ $=0.1-0.4$. The results of infrared spectroscopy showed the

\footnotetext{
a) Author to whom correspondence should be addressed. Tel.: +302107273828; Fax: +30-2107273794. Electronic mail: eikam@eie.gr
}

effect of bulk stoichiometry and oxygen pressure on the developed thin films, which were found to exhibit more polymerized (i.e., cross-linked) germanate networks when prepared under elevated oxygen pressure. It was also noted earlier that the oxygen pressure affects the optical properties of lead-germanate thin films. ${ }^{11}$

In the present work, we report the results of a systematic study of the effects of substrate temperature and oxygen pressure on the optical properties of lead-germanate thin films fabricated by PLD from bulk glassy targets of composition $0.4 \mathrm{PbO}-0.6 \mathrm{GeO}_{2}$. An accurate description of the film structure was achieved by comparing measured infrared transmittance spectra with those calculated using the complex optical functions of the bulk glass and substrate. This study shows that the composition of PLD films can be adjusted by varying the oxygen pressure in the deposition chamber, while their surface quality and optical properties can be improved by controlling the substrate temperature.

\section{EXPERIMENT}

\section{A. Sample preparation}

Bulk glasses in the system $x \mathrm{PbO}-(1-x) \mathrm{GeO}_{2}(x=0.3$, 0.4 , and 0.5 ) were prepared by conventional melting and casting the melts in stainless steel molds, as described earlier. ${ }^{15}$ Cylindrical glass specimens of $15 \mathrm{~mm}$ in diameter and approximately $1 \mathrm{~mm}$ in thickness were prepared and subsequently annealed at $10{ }^{\circ} \mathrm{C}$ below glass transition temperature and polished on both sides.

Lead-germanate thin films were deposited from the bulk glass composition $0.4 \mathrm{PbO}-0.6 \mathrm{GeO}_{2}$ in a stainless steel vacuum chamber, which was evacuated to a residual pressure of $10^{-6}$ mbar prior to each experiment. A $\mathrm{KrF}$ nanosecond excimer laser $(248 \mathrm{~nm})$ operating at $10 \mathrm{~Hz}$ was used for ab- 
lation of the bulk glassy target. A focusing quartz lens with $50 \mathrm{~cm}$ focal length was placed outside the vacuum chamber, and the incidence angle of the laser beam on the target surface was set at $45^{\circ}$. The laser energy fluence incident on the target surface was fixed at $3 \mathrm{~J} / \mathrm{cm}^{2}$. On total, 17500 laser pulses were applied for each deposition. To avoid fast drilling or damage of surface morphology upon laser irradiation, the lead-germanate target was mounted on a vacuum compatible computer-controlled $X Y$ translator. The movement of the translator allowed for the laser spot to scan both vertically and horizontally the surface of the target, leading to a uniformly ablated surface of $10 \mathrm{~mm}^{2}$.

Thin films of approximately $15 \mathrm{~mm}$ in diameter were deposited on infrared-transparent silicon wafers (100 orientations, $0.6 \mathrm{~mm}$ in thickness) having both surfaces optically polished. The substrate was positioned in a furnace allowing for temperature variations from room temperature up to $400{ }^{\circ} \mathrm{C}$, and it was fixed parallel to the target at a distance of $50 \mathrm{~mm}$. The laser ablation was performed under pure oxygen $(99.998 \%)$, and the oxygen pressure in the chamber ranged from $P\left(\mathrm{O}_{2}\right)=0.1$ mbar to $P\left(\mathrm{O}_{2}\right)=0.8$ mbar.

\section{B. Thin film characterization}

The roughness of the deposited films was evaluated using a profilometer (KLA-Tencor Alpha Step500IQ). UV-vis near infrared transmission and reflection spectra (quasinormal reflection at $7^{\circ}$ ) were recorded in the range of $200-1500 \mathrm{~nm}$ at room temperature using a double beam spectrophotometer (Perkin-Elmer Lambda 19). Infrared measurements were performed at room temperature on a Fouriertransform vacuum spectrometer (Bruker 113v), equipped with two detectors and five different beam splitters to cover the frequency range from 30 to $5000 \mathrm{~cm}^{-1}$. Each transmittance or reflectance spectrum resulted from the average of 400 scans. For bulk glasses, the spectra were acquired in the reflectance mode under quasinormal incidence $\left(11^{\circ}\right)$ and $2 \mathrm{~cm}^{-1}$ resolution. For thin films, transmittance spectra were measured at low resolution $\left(10 \mathrm{~cm}^{-1}\right)$ in order to average experimentally the interference pattern due to multiple internal reflections in the silicon substrate.

\section{Spectral simulations}

To reveal the effects of substrate temperature and oxygen pressure on the compositional and structural properties of $\mathrm{Pb}$-germanate thin films, a systematic comparison was made between the infrared spectra measured for PLD thin films and corresponding spectra calculated using the infrared response $(n, k)$ of bulk glassy targets and $\mathrm{Si}$ substrate. To this aim, we made use of a model based on rigorous expressions for transmittance and reflectance of a film/substrate bilayer system which takes into account all optical effects occurring in such a system. We outline below key aspects of the model relevant to the present study, while its detailed description is reported elsewhere. ${ }^{16}$

Each layer is characterized by its thickness $d_{j}$ and complex refractive index $\widetilde{n}_{j}=n_{j}-i k_{j}$ ( $j=1$ for thin film, $j=2$ for substrate, and $j=0,3$ for vacuum), while the phase change of the light beam traversing one layer is given by

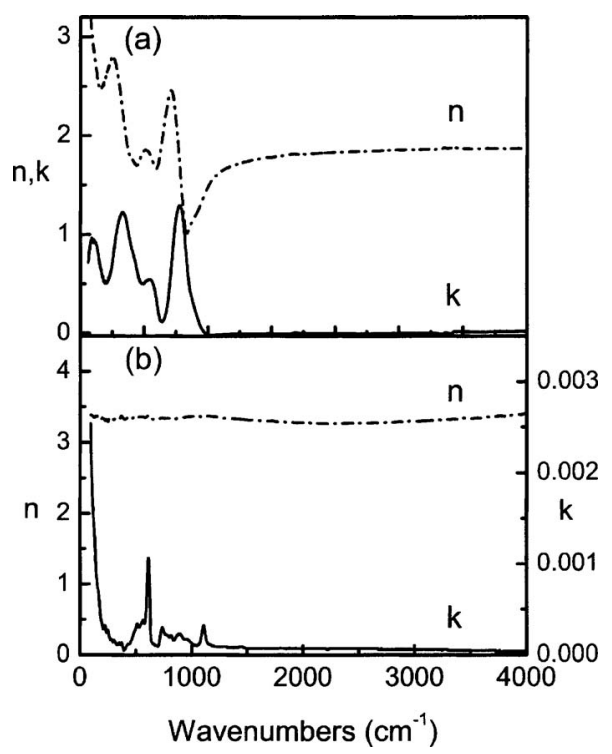

FIG. 1. Real $n$ and imaginary $k$ parts of the complex refractive index for bulk $0.4 \mathrm{PbO}-0.6 \mathrm{GeO}_{2}$ glass (a) and for silicon substrate (b) used for thin film preparation by PLD (for details, see text).

$\delta_{j}=(2 \pi / \lambda) \tilde{n}_{j} d_{j}$, where $\lambda$ is the wavelength in vacuum. The equation for transmittance $T$ of the bilayer system can be written as

$$
T=\frac{t_{1}^{2} t_{2}^{2} t_{3}^{2} e^{-2\left(\gamma_{1}+\gamma_{2}\right)}}{A+B \cos \left(2 \theta_{2}\right)+C \sin \left(2 \theta_{2}\right)},
$$

where $t_{j}=2 \widetilde{n}_{j-1} /\left(\widetilde{n}_{j-1}+\widetilde{n}_{j}\right)$ (denoting the complex transmission Fresnel coefficient at each interface), $\theta_{j}=2 \pi n_{j} d_{j} / \lambda\left(n_{j}\right.$ and $d_{j}$ denote the real part of the refractive index and the thickness, respectively, of layers 1 or 2$), \gamma_{j}=(2 \pi / \lambda) k_{j} d_{j}\left(k_{j}\right.$ is the imaginary part of the refractive index of layers 1 or 2 ), and the terms $A, B$, and $C$ are functions of the thickness $(d)$, the real $(n)$ and imaginary $(k)$ parts of the refractive index of thin film and substrate as given analytically in Ref. 16. As it can be easily shown, the interference fringes due to the $\mathrm{Si}$ substrate used in this study $\left(d_{2}=0.6 \mathrm{~mm}\right)$ have a period of approximately $2.5 \mathrm{~cm}^{-1}$, and, thus, the $\mathrm{Si}$ interference pattern can be optically averaged by measuring low-resolution spectra (e.g., $10 \mathrm{~cm}^{-1}$ ). In this case, Eq. (1) must be averaged with respect to the interference fringes of the substrate in order to compare directly calculated spectra with experimental low-resolution measurements. Integration of Eq. (1) over variable $\theta_{2}$ leads to the substrate interference-free equation for the transmittance $T_{\text {av }}$ of the bilayer system,

$$
T_{\mathrm{av}}=\frac{t_{1}^{2} t_{2}^{2} t_{3}^{2} e^{-2\left(\gamma_{1}+\gamma_{2}\right)}}{S},
$$

where $S=\sqrt{A^{2}+B^{2}+C^{2}}$

In order to compare the spectral characteristics of thin films with those of the target bulk glass, the complex refractive indices of the bulk glass and of the Si substrate are required as input to Eq. (2), with the film thickness being the only adjustable parameter. The measured specular reflectance spectra of bulk glasses were analyzed by Kramers-Kronig transformation to obtain the real $n$ and imaginary $k$ parts of the refractive index of the bulk glass, ${ }^{17}$ as shown in Fig. 1(a) 


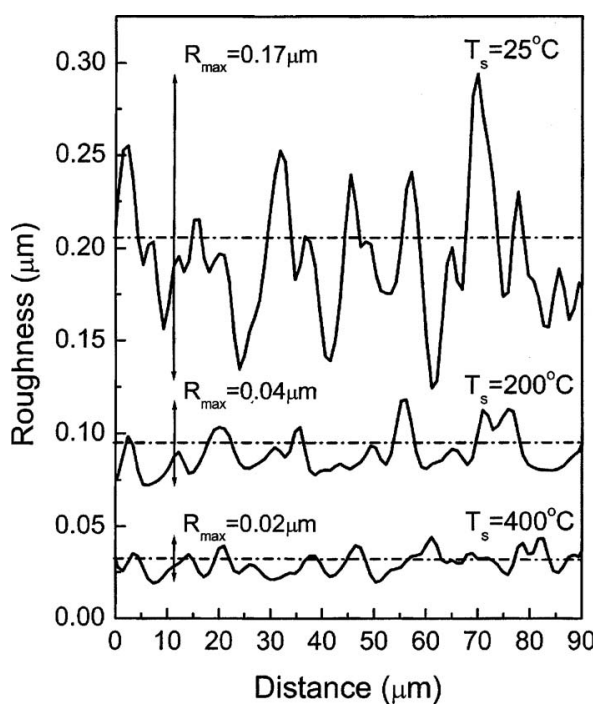

FIG. 2. Roughness of PLD thin films deposited from the $0.4 \mathrm{PbO}-0.6 \mathrm{GeO}_{2}$ glass target on Si substrates of different temperatures $\left(T_{s}\right)$ under the same $\mathrm{O}_{2}$ pressure $(0.45 \mathrm{mbar}) . R_{\max }$ represents the maximum difference between the highest and the lowest point in the probe surface area. Roughness data are offset to facilitate comparison.

for the bulk $0.40 \mathrm{PbO}-0.6 \mathrm{GeO}_{2}$ glass. The infrared properties of the Si substrate are shown in Fig. 1(b), as determined previously ${ }^{16}$ and confirm its transparency in the infrared range. The bands observed in the $k$ spectrum at 565,608 , $740,815,890,965,1302$, and $1450 \mathrm{~cm}^{-1}$ originate from lattice modes of $\mathrm{Si}^{18}{ }^{18}$ while the feature at $1107 \mathrm{~cm}^{-1}$ indicates oxygen impurities as arising from the asymmetric stretching vibration of $\mathrm{Si}-\mathrm{O}-\mathrm{Si}$ bridges. ${ }^{19}$ Comparison in Fig. 1 of the $k$ spectra of glass and $\mathrm{Si}$ substrate shows that all bands of $\mathrm{Si}$ are weak relative to those of the $\mathrm{Pb}$-germanate glass.

\section{RESULTS}

\section{A. Influence of substrate temperature}

Results of profilometry measurements are shown in Fig. 2 for three typical $\mathrm{Pb}$-germanate films deposited from the $0.4 \mathrm{PbO}-0.6 \mathrm{GeO}_{2}$ glassy target under the same oxygen pressure $(0.45$ mbar) but with different substrate temperatures. It is clearly observed that the surface roughness of the PLD film improves remarkably when the substrate temperature increases from 25 to $400{ }^{\circ} \mathrm{C}$.

Along these lines, it is of interest to explore also the corresponding effect on the optical response of thin films in the 200-1500 nm spectral range. Transmission measurements show that the absorption edges of the Si substrate and of the target bulk glass are at 1010 and $345 \mathrm{~nm}$, respectively, while the reflectivity of Si remains quite high in the entire spectral range, Fig. 3(a). Thus, the PLD films on Si were measured in the specular reflectivity mode and the recorded spectra are shown in Fig. 3(b). When the substrate is heated at $400{ }^{\circ} \mathrm{C}$ during the deposition, well-defined interference fringes are observed due to multiple, reflection in the thin film. This interference pattern appears superimposed on the Si reflectivity for wavelengths above $400 \mathrm{~nm}$ and tends to disappear for wavelengths below the absorption edge of the bulk glass. Similar observations are made for the film depos-

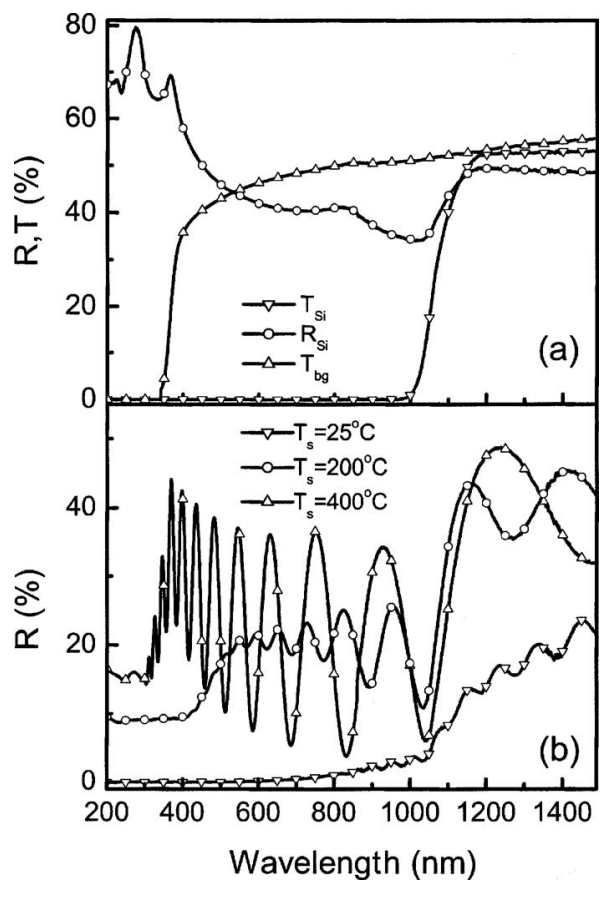

FIG. 3. (a) Optical reflectivity $R_{\mathrm{Si}}$ and transmittance $T_{\mathrm{Si}}$ of the silicon substrate $(d=0.6 \mathrm{~mm})$ and the $0.4 \mathrm{PbO}-0.6 \mathrm{GeO}_{2}$ bulk glass, $T_{\mathrm{bg}}(d=1 \mathrm{~mm})$, and (b) reflectivity of corresponding thin films deposited under different substrate temperatures, $T_{s}$ and an $\mathrm{O}_{2}$ pressure of $0.45 \mathrm{mbar}$.

ited at substrate temperature $T_{s}=200{ }^{\circ} \mathrm{C}$. However, the amplitude of fringes is now reduced and the reflectivity falls below $600 \mathrm{~nm}$, which is well above the absorption edge of the bulk glass. The reflectivity of the room temperature PLD film is low compared to the other two films, and its value diminishes below $1100 \mathrm{~nm}$. The degraded optical response of the $T_{s}=25^{\circ} \mathrm{C}$ film can result from light scattering due to its large surface roughness (Fig. 2), manifesting the influence of substrate temperature on the optical properties of PLD films. While surface roughness is shown to have a strong influence on the optical response of the PLD films in the visible, their possible nonstoichiometry in oxygen may also affect the optical properties. A manifestation of such an effect could be the gradual shift of the wavelength at which the reflectivity diminishes as the substrate temperature increases from $T_{s}=25^{\circ} \mathrm{C}$ to $T_{s}=400{ }^{\circ} \mathrm{C}$ [Fig. 3(b)].

In Fig. 4 are presented low-resolution infrared transmittance spectra of thin films deposited under different substrate temperatures, with the infrared measurements having been performed right after film preparation. The spectrum of the film fabricated at $T_{s}=25^{\circ} \mathrm{C}$ exhibits an intense band around $3350 \mathrm{~cm}^{-1}$ associated with the stretching mode of molecular water and a weaker band at $1695 \mathrm{~cm}^{-1}$ due also to water (i.e., bending mode). As no clear interference pattern could be distinguished, no spectral simulation was attempted for this thin film. Thus, even though strong absorption bands associated with the germanate network are observed below $1000 \mathrm{~cm}^{-1}$, the poor optical quality of this film prohibits the quantitative treatment of its infrared spectrum.

The spectra of the other two films present interference patterns in the $1250-4000 \mathrm{~cm}^{-1}$ range, which allows spectral calculations-using the infrared response of the target bulk glass-and comparisons with experimental spectra. Results 


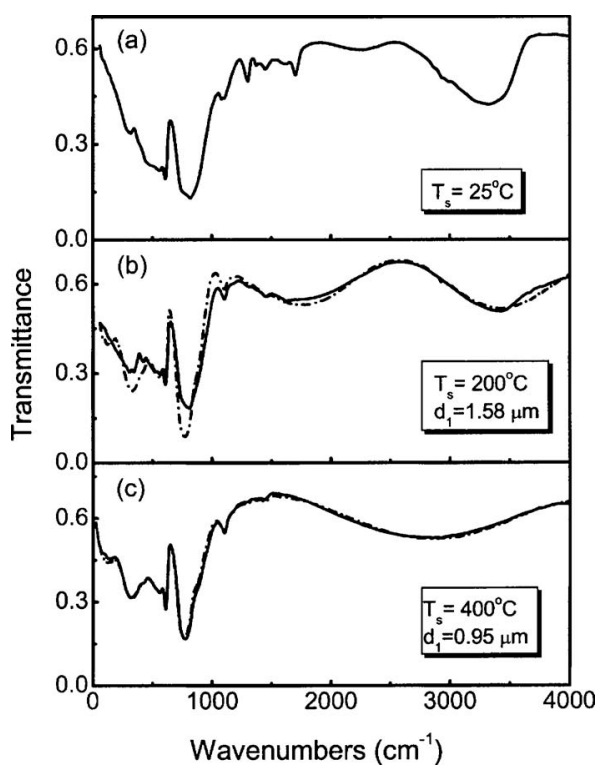

FIG. 4. Measured infrared transmittance spectra (continuous lines) for films deposited at different substrate temperatures $T_{s}$ from the $0.4 \mathrm{PbO}-0.6 \mathrm{GeO}_{2}$ glass target under $\mathrm{O}_{2}$ pressure of $0.45 \mathrm{mbar}$. The dash-dotted lines show calculated spectra employing Eq. (2) and the optical response of the glass target and the Si substrate. The indicated film thickness $\left(d_{1}\right)$ in (b) and (c) is the best value obtained by simulating the experimental spectra.

of spectral simulations performed using Eq. (2) are shown in Fig. 4, where $d_{1}$ is the thin film thickness giving the best agreement between simulated and experimental interference patterns in the frequency range above $1250 \mathrm{~cm}^{-1}$, a range free from absorption bands of both thin film and substrate. For $T_{s}=200{ }^{\circ} \mathrm{C}$, differences are observed between calculated and experimental spectra. These include the signature of water in the experimental spectrum (band at approximately $3400 \mathrm{~cm}^{-1}$ ) and obvious variations in band intensity and shape for bands below $1200 \mathrm{~cm}^{-1}$. The overall agreement between simulation and experiment improves remarkably for the $T_{s}=400{ }^{\circ} \mathrm{C}$ film, while the broad infrared absorption bands point toward the amorphous nature of the film. The close similarity with simulation suggests that the PLD film has chemical composition and network structure similar to those of the parent bulk glass. In addition, no sign of water was detected for PLD film developed at $T_{s}=400{ }^{\circ} \mathrm{C}$. Thus, we focus now our analysis on thin films developed at $400{ }^{\circ} \mathrm{C}$.

Considering the tendency of films made at lower substrate temperatures to absorb water after removal from the vacuum chamber, we checked the corresponding stability of the film deposited at $T_{s}=400{ }^{\circ} \mathrm{C}$. As shown in the spectra of Fig. 5, exposure to air for 4 months causes no detectable absorption of water by this high temperature film. Some spectral differences concerning the amplitude and period of interference fringes and the intensity of germanate bands (e.g., at $778 \mathrm{~cm}^{-1}$ ) arise from small thickness differences between the probed film spots before and after exposure to air. These findings stress again the importance of the substrate temperature on the stability of amorphous lead-germanate films deposited by PLD.

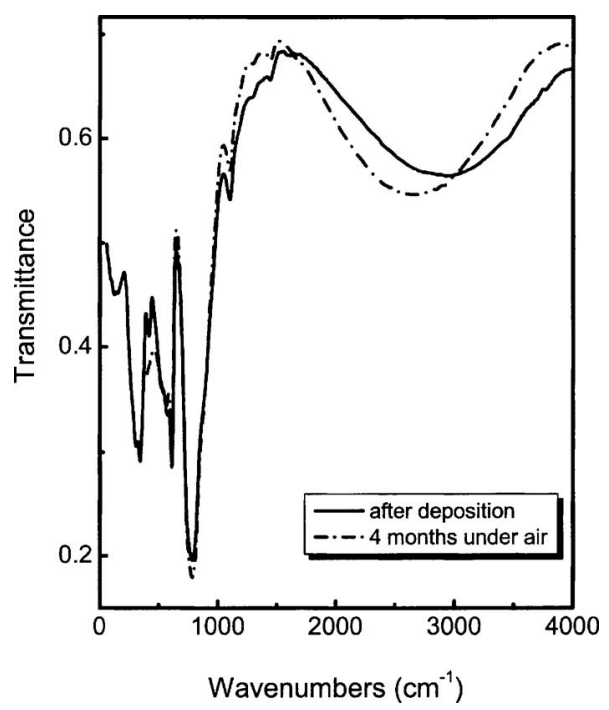

FIG. 5. Infrared transmittance spectra of a thin film deposited from the $0.4 \mathrm{PbO}-0.6 \mathrm{GeO}_{2}$ glass target under $0.45 \mathrm{mbar} \mathrm{O}_{2}$ pressure and $T_{s}$ $=400{ }^{\circ} \mathrm{C}$ substrate temperature. The continuous-line spectrum was measured right after deposition and the dash-dotted line spectrum after 4 months of film exposure to air.

\section{B. Influence of oxygen pressure}

Having established the key role of substrate temperature, we examine now the role of oxygen pressure on films prepared at $T_{s}=400{ }^{\circ} \mathrm{C}$. Typical low-resolution transmittance spectra measured for films deposited under oxygen pressure ranging from 0.1 to 0.8 mbar are depicted in Fig. 6. The results of simulations, using again Eq. (2) and the optical properties of the bulk $0.4 \mathrm{PbO}-0.6 \mathrm{GeO}_{2}$ glass and of the $\mathrm{Si}$ substrate, show good agreement with the experimental spectra in the entire spectral range. The very good matching in the "transparent parts" of the spectra (approximately $1500-4000 \mathrm{~cm}^{-1}$ range) allows us to probe the effect of oxygen pressure on the vibrational bands of films

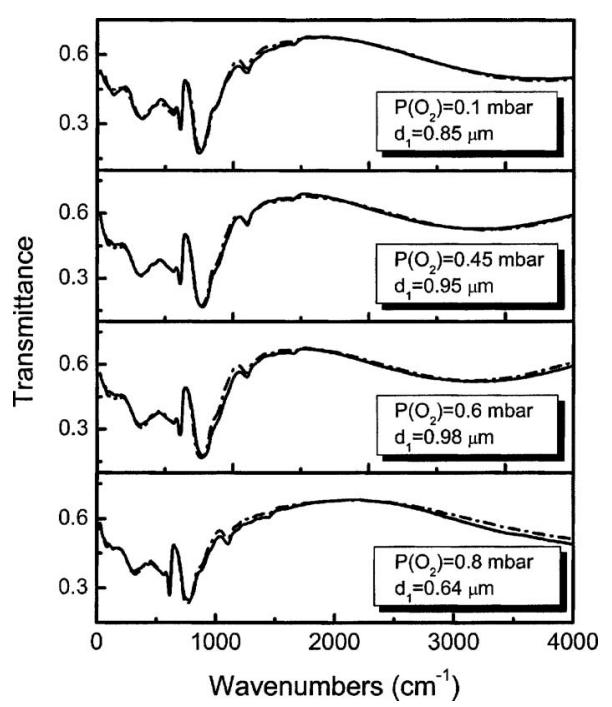

FIG. 6. Infrared transmittance spectra (continuous lines) of films deposited from the $0.4 \mathrm{PbO}-0.6 \mathrm{GeO}_{2}$ glass target under the same substrate temperature $\left(T_{s}=400{ }^{\circ} \mathrm{C}\right)$ and different $\mathrm{O}_{2}$ pressures $P\left(\mathrm{O}_{2}\right)$. Dotted-line spectra were calculated using Eq. (2) and the optical response of the glass target and the Si substrate. The best-fitted film thickness is indicated by $d_{1}$. 


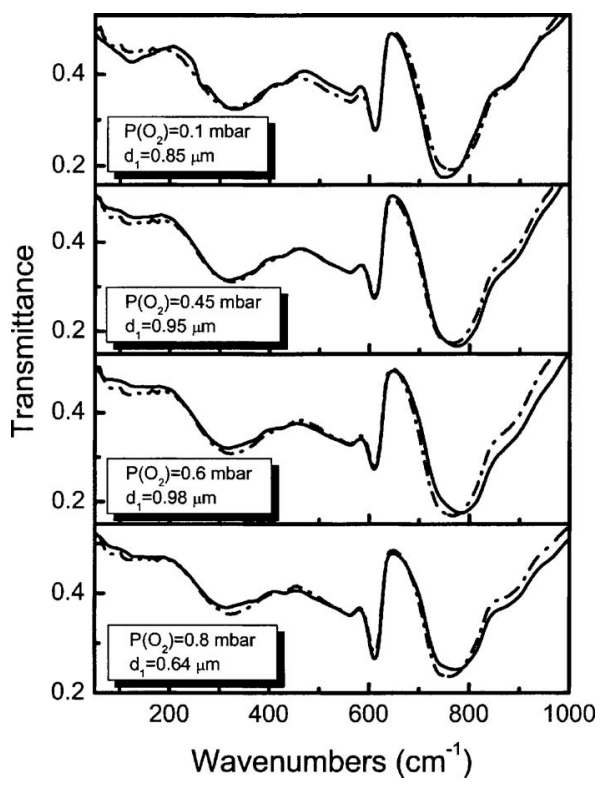

FIG. 7. The infrared spectra of Fig. 6 presented in an expanded frequency scale to highlight the effect of oxygen pressure on the vibrational bands of the PLD films.

$\left(50-1200 \mathrm{~cm}^{-1}\right)$. This is done in Fig. 7 where we compare the evolution of the experimental spectra in parallel with those calculated by Eq. (2). In doing so, we do not take into account differences in experimental spectra due to purely optical effects which arise from film thickness variations. ${ }^{20}$

As observed in Fig. 7, the spectrum of the film deposited under $\mathrm{P}\left(\mathrm{O}_{2}\right)=0.1$ mbar shows in comparison to simulation a shift of the $750-780 \mathrm{~cm}^{-1}$ envelop to lower wave numbers, a small shift to higher wave numbers for the $310-330 \mathrm{~cm}^{-1}$ band, and a larger intensity at approximately $120 \mathrm{~cm}^{-1}$. When the oxygen pressure increases above $0.1 \mathrm{mbar}$, the $750-780 \mathrm{~cm}^{-1}$ envelope becomes broader and shifts to higher frequencies, the $310-330 \mathrm{~cm}^{-1}$ band shifts to lower frequencies, and the band below $200 \mathrm{~cm}^{-1}$ in the far-infrared exhibits reduced intensity. In parallel, the weak and asymmetric band peaking at $560 \mathrm{~cm}^{-1}$ tends to gain intensity relative to simulation. It is noted that these spectral trends seem to saturate when the oxygen pressure exceeds 0.6 mbar.

In order to search for the origin of the observed spectral variations for films deposited under different oxygen pressures, we present in Fig. 8 the $k$ spectra of bulk $\mathrm{Pb}-$ germanate glass with lead oxide contents of 50, 40, and $30 \mathrm{~mol} \%$. Although decreasing lead oxide content in Fig. 8 causes spectral trends more pronounced than those exhibited by thin films deposited at increasing oxygen pressure (Fig. 7), both trends are in the same direction.

The observed evolutions of the infrared transmittance spectra of thin films, deposited either under different oxygen pressures or different substrate temperatures, point toward the systematic influence of the deposition parameters on the compositional and/or structural characteristics of the PLD films. Possible explanations for the present findings will be discussed in the following section.

\section{DISCUSSION}

It was found in this study that increasing substrate temperature during deposition leads to substantial improvements

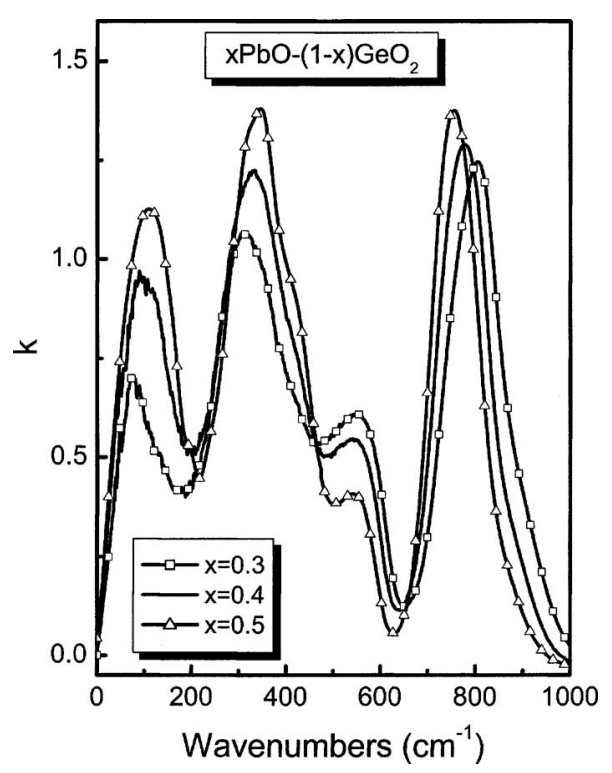

FIG. 8. Extinction coefficient $k$ spectra of bulk $x \mathrm{PbO}-(1-x) \mathrm{GeO}_{2}$ glasses with different mole fractions $x$ of lead oxide.

in surface quality (Fig. 2), optical response [Fig. 3(b)], and stability (Figs. 4 and 5) of the pulsed-laser deposited $\mathrm{Pb}$ germanate thin films. The present results are in agreement with previous reports on the effect of substrate temperature on the morphology and structure of deposited films. ${ }^{21-24}$ These studies have emphasized the influence of substrate temperature on the mobility of adatoms constituting the growing film surface, with the specific effect of temperature depending on the nature of the deposited material. For the present study, the PLD material is amorphous and the highest substrate temperature employed is $20{ }^{\circ} \mathrm{C}$ below the glass transition temperature of the bulk Pb-germanate target. ${ }^{25}$ Thus, for $T_{s}=400{ }^{\circ} \mathrm{C}$ the growth of film is accompanied by annealing with no signs of crystallization detectable by infrared spectroscopy. It is suggested that this parallel annealing process enables atoms in the film to migrate and rearrange in energetically favorable local sites. This leads eventually to surface energy minimization and to improvement of surface texture and quality, and, consequently, to enhanced film durability toward water vapors.

Before considering the influence of oxygen pressure on films developed at constant substrate temperature, $T_{s}$ $=400{ }^{\circ} \mathrm{C}$, it is instructive to refer to the origin of the main absorption bands in Fig. 7. They appear at 750-780, 560, 310-330, and approximately $120 \mathrm{~cm}^{-1}$, besides the Si band at $608 \mathrm{~cm}^{-1}$. Earlier midinfrared ${ }^{25}$ and extended $\mathrm{x}$-ray absorption fine structure ${ }^{26}$ studies on bulk $x \mathrm{PbO}-(1-x) \mathrm{GeO}_{2}$ glasses have suggested a progressive depolymerization of the germanate network upon increasing $\mathrm{PbO}$ content. This process results from the breaking of $\mathrm{Ge}-\mathrm{O}-\mathrm{Ge}$ bridges and the creation of nonbridging oxygen (NBO) atoms on $Q^{n}$ germanate tetrahedral units with 4- $n$ NBOs per tetrahedron. According to this process, the network of the bulk $0.4 \mathrm{PbO}-0.6 \mathrm{GeO}_{2}$ glass would be dominated by $Q^{3}$ and $Q^{2}$ tetrahedral units, with minor contributions from $Q^{4}$ and $Q^{1}$ units. ${ }^{27}$ On these grounds, and following infrared assignments for alkali germanate glasses, ${ }^{27,28}$ we attribute the 


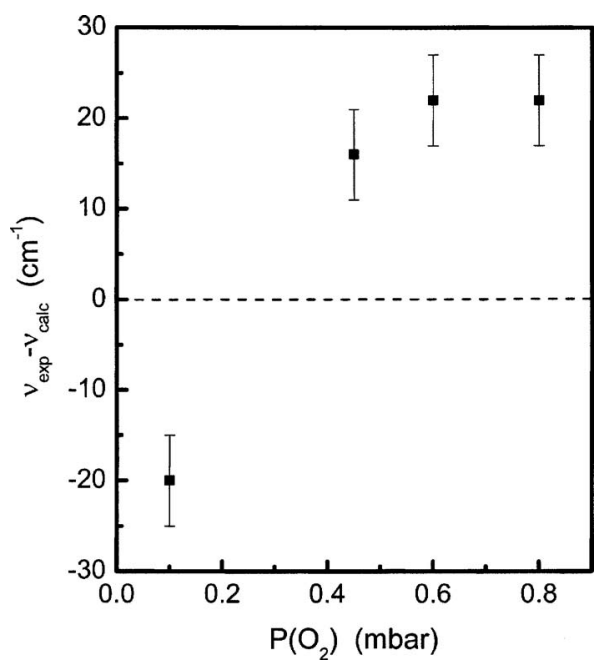

FIG. 9. Effect of oxygen pressure $P\left(\mathrm{O}_{2}\right)$ on the germanate network of PLD films grown from the $0.4 \mathrm{PbO}-0.6 \mathrm{GeO}_{2}$ glass target at a constant substrate temperature $\left(T_{s}=400{ }^{\circ} \mathrm{C}\right)$. Frequencies $\nu_{\text {exp }}$ and $\nu_{\text {calc }}$ correspond to frequencies in Fig. 7, where the experimental and simulated spectra, respectively, show their minimum transmittance in the $750-780 \mathrm{~cm}^{-1}$ spectral range. For more details, see text.

strong $750-780 \mathrm{~cm}^{-1}$ profile to convolution of different modes. These include the asymmetric stretching vibration of $\mathrm{Ge}-\mathrm{O}-\mathrm{Ge}$ bridges contributing above $800 \mathrm{~cm}^{-1}$ and the asymmetric stretching vibration of $Q^{n}$ germanate tetrahedral with NBOs appearing below $800 \mathrm{~cm}^{-1}$ (with frequency decreasing upon decreasing $n$ ). The lower-intensity band at $560 \mathrm{~cm}^{-1}$ arises from the bending of $\mathrm{Ge}-\mathrm{O}-\mathrm{Ge}$ bridges and the broad band at $310-330 \mathrm{~cm}^{-1}$ from the rocking motion of oxygen atoms in $\mathrm{Ge}-\mathrm{O}-\mathrm{Ge}$ bridges. The far-infrared band around $120 \mathrm{~cm}^{-1}$ can be attributed to $\mathrm{Pb}-\mathrm{O}$ stretching, in accordance with earlier far-infrared studies on metal-oxide containing glasses. ${ }^{29}$ The relatively weak intensity of the $120 \mathrm{~cm}^{-1}$ band indicates a considerable degree of covalent character in the $\mathrm{Pb}-\mathrm{O}$ bond, in agreement with a complementary strong activity measured at $105-130 \mathrm{~cm}^{-1}$ in the Raman spectra of $\mathrm{Pb}$-germanate glasses. ${ }^{30}$

On the basis of the above assignments, and the observed spectral trends exhibited by thin films (Fig. 7) and bulk $\mathrm{Pb}$ germanate glasses (Fig. 8), we suggest that higher oxygen pressures in the deposition chamber lead to films with progressively increasing polymerization of their germanate network, i.e., with decreasing lead oxide content. To present schematically such an evolution of the germanate network we plot in Fig. 9 the frequency difference $\nu_{\text {exp }}-\nu_{\text {calc }}$ as a function of oxygen pressure, where $\nu_{\exp }$ and $\nu_{\text {calc }}$ are the frequencies at which the experimental and simulated spectra, respectively, show their minimum transmittance in the $750-780 \mathrm{~cm}^{-1}$ range. As observed in Fig. 9, the difference between experimental and simulated spectra shows its largest change for oxygen pressures in the range $P\left(\mathrm{O}_{2}\right)$ $=0.1-0.6 \mathrm{mbar}$, and it tends to stabilize at higher oxygen pressures.

The spectral comparisons presented in Fig. 10 allow us to gain additional information for the effect of oxygen pressure on the composition of PLD thin films. The experimental spectra of two films made from the $0.4 \mathrm{PbO}-0.6 \mathrm{GeO}_{2}$ glass

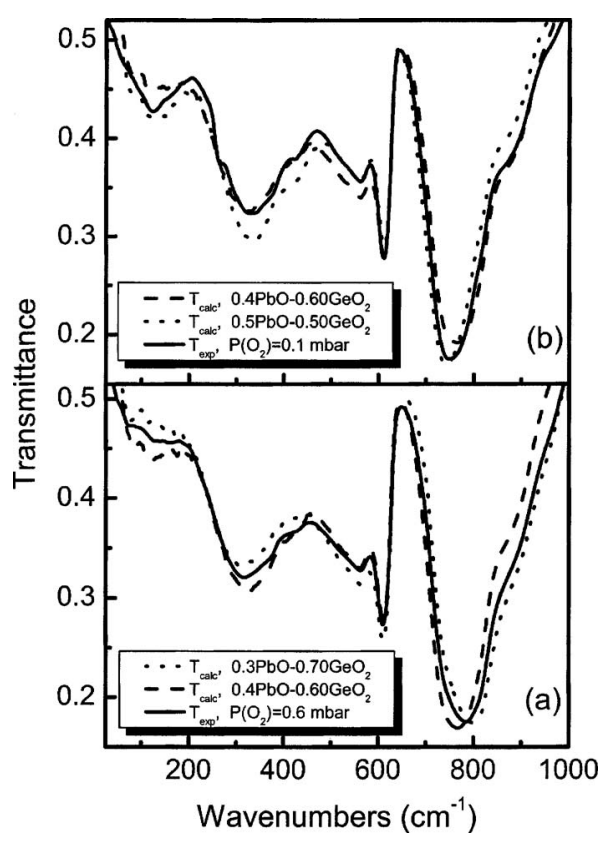

FIG. 10. Comparison of experimental infrared transmittance spectra of PLD films, deposited from the $0.4 \mathrm{PbO}-0.6 \mathrm{GeO}_{2}$ glass target at $T_{s}=400{ }^{\circ} \mathrm{C}$ and oxygen pressures $P\left(\mathrm{O}_{2}\right)=0.6$ mbar (a) and 0.1 mbar (b), with those calculated using the optical constants of bulk $x \mathrm{PbO}-(1-x) \mathrm{GeO}_{2}$ glasses with different lead oxide contents $(x=0.3,0.4$, and 0.5$)$.

target, under constant substrate temperature $\left(T_{s}=400{ }^{\circ} \mathrm{C}\right)$ and $P\left(\mathrm{O}_{2}\right)=0.1$ and 0.6 mbar, are compared with spectra calculated using the infrared responses of the $x=0.3,0.4$, and 0.5 bulk germanate glasses $x \mathrm{PbO}-(1-x) \mathrm{GeO}_{2}$. As above, the film thickness in simulations is that giving the best agreement between experiment and simulation in the "transparent" spectral range of $1500-5000 \mathrm{~cm}^{-1}$. As shown in Fig. 10(a), the spectral profile of the $P\left(\mathrm{O}_{2}\right)=0.6 \mathrm{mbar}$ thin film is between those simulated for bulk compositions $x=0.4$ and $x$ $=0.3$. Thus, band shapes and relative intensities indicate that this film should have lead oxide content between those of the bulk glassy target $x=0.4$ and the $x=0.3$ bulk glass. Similarly, the spectra presented in Fig. 10(b) suggest for the $P\left(\mathrm{O}_{2}\right)$ $=0.1 \mathrm{mbar}$ thin film a lead oxide content higher than that of the target $(x=0.4)$ but lower than $x=0.5$. Therefore, the composition of the PLD Pb-germanate film can be adjusted by varying the oxygen pressure in the deposition chamber.

The effect of oxygen pressure on the composition of $\mathrm{Pb}$-germanate films can be viewed in terms of the interactions between ablated atoms and oxygen in the deposition chamber. Earlier studies of the laser-produced plasma in PLD experiments have suggested two key interaction regimes between the background gas and the ablated atoms; the elastic scattering and the reactive collision regimes. ${ }^{31}$ If we consider the first interaction regime to be dominant, then mass considerations suggest that the effect on lead would be less pronounced than for germanium atoms. Thus, one would expect the relative content of lead in the films to increase with oxygen pressure due to increased elastic scattering interactions. Since this is opposite to the trends found in this study, it appears that differences in reactivity of lead and germanium with oxygen in the plume should play a predominant role for the observed variations in film composition. As 
shown by infrared spectroscopy, composition variations are more profound for oxygen pressures in the range of 0.1-0.6 mbar and tend to stabilize at higher oxygen pressures. Thus, we suggest that the larger reactivity of germanium with oxygen atoms drives the PLD film composition in the range $P\left(\mathrm{O}_{2}\right)=0.1-0.6$ mbar. At higher oxygen pressures both germanium and lead atoms react fully with oxygen and, thus, contribute toward stabilization of film composition. Nevertheless, differences in composition between films and target remain even at high oxygen pressures and they may be correlated with differences in evaporation rates of lead and germanium. In conclusion, this study has demonstrated the possibility to control the composition of PLD lead-germanate thin films by changing the oxygen pressure during deposition.

\section{CONCLUDING REMARKS}

Amorphous lead-germanate thin films were successfully fabricated on silicon substrates by pulsed-laser deposition of the bulk $0.4 \mathrm{PbO}-0.6 \mathrm{GeO}_{2}$ glassy target. Infrared transmittance measurements allowed the systematic study of the effects of substrate temperature and oxygen pressure on the deposited films by probing the evolution of their structural characteristics. Substrate temperature of $400{ }^{\circ} \mathrm{C}$ was found to improve the optical transparency and stability of the deposited thin films. When lower substrate temperatures were employed, the optical quality and durability of films were found to decrease drastically, and these changes were correlated with the surface roughness of the deposited films. Variations in composition of films deposited at the optimum substrate temperature, $400{ }^{\circ} \mathrm{C}$, and under oxygen pressures from 0.1 to 0.8 mbar were tracked by comparing measured infrared transmittance spectra with corresponding calculated spectra. Calculations were made by using rigorous expressions for transmittance and the infrared response of the $\mathrm{Si}$ substrate and of bulk glassy compositions $x \mathrm{PbO}-(1$ $-x) \mathrm{GeO}_{2}$ with $x=0.3,0.4$, and 0.5 . The results showed that the lead oxide content of the deposited $\mathrm{Pb}$-germanate films decreases when the oxygen pressure varies from 0.1 to 0.6 mbar, and this leads to films with more polymerized germanate networks. These phenomena were found to stabilize at higher oxygen pressures, and they were discussed in terms of differences in reactivity of lead and germanium with oxygen in the laser-produced plasma.

\section{ACKNOWLEDGMENT}

Partial financial support of this work by the EU through the Marie Curie Actions-NANONLO project (MTKD-CT2006-042301) is gratefully appreciated.

\footnotetext{
${ }^{1}$ L. Tong, R. R. Gattas, I. Maxwell, J. B. Ashcom, and E. Mazur, Opt. Commun. 259, 626 (2006).

${ }^{2}$ Q. Y. Zhang, K. Pita, C. K. F. Ho, N. Q. Ngo, L. P. Zuo, and S. Takahashi,
}

Chem. Phys. Lett. 368, 183 (2003).

${ }^{3}$ R. Nayak, V. Gupta, A. L. Dawar, and K. Sreenivas, Thin Solid Films 445, 118 (2003).

${ }^{4}$ R. Serna, C. N. Afonso, J. M. Ballesteros, and A. Zschocke, Appl. Surf. Sci. 109/110, 524 (1997).

${ }^{5}$ A. Pillonnet, C. Garapon, C. Champeaux, C. Bovier, H. Jaffrezic, and J. Mugnier, J. Lumin. 87-89, 1087 (2000).

${ }^{6}$ M. Martino, A. P. Caricato, M. Fernandez, G. Leggieri, M. Ferrari, and M. Mattarelli, Thin Solid Films 433, 39 (2003).

${ }^{7}$ M. Dussauze, A. Malakho, E. Fargin, J. P. Manaud, V. Rodriguez, F. Adamietz, and B. Lazoriak, J. Appl. Phys. 100, 13108 (2006).

${ }^{8}$ B. Ferreira, E. Fargin, J. P. Manaud, G. Le Flem, V. Rodriguez, and T. Buffeteau, J. Non-Cryst. Solids 343, 121 (2004).

${ }^{9}$ Y. Luo, A. Biswas, A. Frauenglass, and S. R. J. Brueck, Appl. Phys. Lett. 84, 4935 (2004).

${ }^{10}$ S. Mailis, A. A. Anderson, A. J. Barrington, W. S. Brocklesvy, R. Greef, H. N. Rutt, R. W. Eason, N. A. Vainos, and C. Grivas, Opt. Lett. 23, 1751 (1999)

${ }^{11}$ S. Mailis, C. Riziotis, J. Wang, E. Taylor, A. A. Anderson, S. J. Barrington, H. N. Rutt, R. W. Eason, N. A. Vainos, and C. Grivas, Opt. Mater. (Amsterdam, Neth.) 12, 27 (1999).

${ }^{12}$ W. A. Pliskin and H. S. Lehman, J. Electrochem. Soc. 112, 1013 (1965); N. Nakamura, Y. Mochizuki, K. Usami, Y. Itoh, and T. Nozaki, Solid State Commun. 50, 1079 (1984).

${ }^{13}$ R. R. Koropecki, R. Arce, L. S. Bernardez, and R. Buitrago, J. NonCryst. Solids 74, 11 (1985); F. Geotti-Bianchini, L. Riu, G. Gagliardi, M. Guglielmi, and C. G. Pantano, Glastech. Ber. 64, 205 (1991).

${ }^{14}$ A. Morimoto, H. Noriyama, and T. Shimizu, Jpn. J. Appl. Phys., Part 1 26, 22 (1987)

${ }^{15}$ A. Tsigara, L. Velli, A. Giannoudakos, C. P. E. Varsamis, M. Kompitsas, N. A. Vainos, and E. I. Kamitsos, Appl. Phys. A: Mater. Sci. Process. 79, 1319 (2004)

${ }^{16}$ E. I. Kamitsos, M. Dussauze, C. P. E. Varsamis, P. Vinatier, and Y. Hamon, J. Phys. Chem. C. 111, 8111 (2007).

${ }^{17}$ E. I. Kamitsos, A. P. Patsis, M. A. Karakassides, and G. D. Chryssikos, J. Non-Cryst. Solids 126, 52 (1990).

${ }^{18}$ I. V. Boyd and J. I. B. Wilson, J. Appl. Phys. 53, 4166 (1982).

${ }^{19}$ E. I. Kamitsos, Phys. Rev. B 53, 14659 (1996).

${ }^{20}$ C. P. E. Varsamis, E. I. Kamitsos, and G. D. Chryssikos, Phys. Rev. B 60, 3885 (1999)

${ }^{21}$ E. Lopez, S. Chiussi, P. Gonzalez, J. Serra, and B. Leon, Appl. Surf. Sci. 248, 108 (2005).

${ }^{22}$ B. Borer, A. Sonnenfeld, and P. R. von Rohr, Surf. Coat. Technol. 201, 1757 (2006).

${ }^{23}$ H. Xia, S. B. Tang, L. Lu, Y. S. Meng, and G. Ceder, Electrochim. Acta 52, 2822 (2007).

${ }^{24}$ Y. S. Jung, Appl. Surf. Sci. 221, 281 (2004).

${ }^{25}$ J. E. Canale, R. A. Condrate, K. Naussau, and B. S. Cornilsen, J. Can. Ceram. Soc. 55, 50 (1986).

${ }^{26}$ S. J. L. Ribeiro, J. Dexpert-Ghys, B. Piriou, and V. R. Mastelaro, J. Non-Cryst. Solids 159, 213 (1993).

${ }^{27}$ E. I. Kamitsos, Y. D. Yiannopoulos, C. P. Varsamis, and H. Jain, J. NonCryst. Solids 222, 59 (1997).

${ }^{28}$ E. I. Kamitsos, Y. D. Yiannopoulos, M. A. Karakassides, G. D. Chryssikos, and H. Jain, J. Phys. Chem. 100, 11755 (1996); P. V. Teredesai, D. T. Anderson, N. Hause, K. Lantezky, and J. L. Yarger, Phys. Chem. Glasses 40, 345 (2005).

${ }^{29}$ P. Tarte, A. Rulmont, and M. Almou, Phys. Chem. Glasses 28, 130 (1987); E. I. Kamitsos, Y. D. Yiannopoulos, H. Jain, and W. C. Huang, Phys. Rev. B 54, 9775 (1996); E. I. Kamitsos and G. D. Chryssikos, Solid State Ionics 105, 75 (1998).

${ }^{30}$ V. N. Sigaev, I. Gregora, P. Pernice, B. Champagnon, E. N. Smelyanskaya, A. Aronne, and P. D. Sarkisov, J. Non-Cryst. Solids 279, 136 (2001).

${ }^{31}$ X. Y. Chen, S. B. Xiong, Z. S. Sha, and Z. G. Liu, Appl. Surf. Sci. 115, 279 (1997). 